Canad. Math. Bull. Vol. 20 (1), 1977

\title{
ON THE NON-EXISTENCE OF INJECTIVE NEAR-RING MODULES
}

\author{
BY \\ BERNHARD BANASCHEWSKI AND EVELYN NELSON
}

Associated with any near-ring $R$, and any set $S$ of distributive elements of $R$, one has a natural notion of $S$-distributive $R$-modules, analogous to that of modules over rings and including the latter as special case (Frohlich [6]; we recall the details in Section 1). Since near-rings can be viewed as slightly deficient rings, it makes sense to enquire whether such near-ring modules share with modules over rings the familiar and important property of having injective hulls. The purpose of this paper is to demonstrate that even the unitary, distributively generated near-rings, which may be regarded as nearest to rings, can be very badly unlike rings with respect to their injective modules.

We present examples of such near-rings $R$ for which the category of unitary $S$-distributive $R$-modules (with suitable $S$ ) has no non-trivial absolute retracts and hence no non-trivial injectives, first for the case when $R$ is actually a ring, and second for the case that $S$ is the set of all distributive elements of $R$. A further example of the latter type provides a case in which there are a lot of, but nonetheless not enough, injectives. Our first example, in addition, shows that the familiar Baer criterion which characterizes injectivity of modules fails to do this for near-ring modules, as was mistakenly claimed in Seth-Tewari [9] and has been disproved independently by Oswald [8].

The main tool for obtaining our examples is the concept, due to Fröhlich [6], of the semigroup near-ring $N[A]$ of a semigroup $A$, which is the near-ring counterpart of the familiar notion of semigroup ring. It turns out that for any non-trivial monoid $A$, the category of all $N[A]$-modules does not have enough injectives; in fact, it is not even residually small.

For the general theory of injectivity and related concepts in equationally defined classes of algebras (of which modules over near-rings are special cases) the reader is referred to Banaschewski [1], and for source material on near-rings and their modules to Fröhlich [5] and Beidleman [3].

The results presented here were announced in the AMS Notices 23 (1976) A-8.

1. Preliminaries. A (left) near-ring $R$ is a set with two binary operations, addition $(x, y) \leadsto x+y$ and multiplication $(x, y) \leadsto x y$, such that + is a (not

Received by the editors Jan. 4, 1977. 
necessarily commutative) group operation, with neutral element 0 , and the multiplication is associative and distributes over addition from the left (i.e. $x(y+z)=x y+x z)$.

If there is an element $1 \in R$ with $x 1=1 x=x$ for all $x \in R$ then $R$ is called unitary.

An element $s \in R$ is called distributive if $(x+y) s=x s+y s$ for all $x, y \in R$.

$R$ is said to be distributively generated (d.g. for short) if its additive group is generated by the set $D R$ of all distributive elements of $R$, (Fröhlich [5]). Evidently, $D R$ is a multiplicatively closed subset of $R$ and contains the unit of $R$ if it exists. Note that a d.g. near-ring $R$ whose additive group is commutative is a ring [5].

The canonical example of a near-ring is given by $T(G)$, the near-ring of selfmaps of a group $G$, whose elements are the maps of (the underlying set of) $G$ into itself, with addition defined pointwise in terms of the operation in $G$ and multiplication given by composition. Jacobson $[7, \mathrm{p} .1]$ points out that $T(G)$ is "very nearly a ring"; note, though, that $T(G)$ is actually never a ring for non-trivial $G$, in view of the constant maps. The identity element of $T(G)$ is the identity map of $G$, and its distributive elements are precisely the endomorphisms of $G$. Moreover, the subnear-ring of $T(G)$ generated by any set $S$ of endomorphisms of $G$ which is closed under functional composition is a d.g. near-ring.

For a near-ring $R$ and a subset $S \subset D R$, an $S$-distributive $R$-module $M$, called an $(R, S)$-group in Frölich [6], is a group, together with a family $x \leadsto x r(r \in R)$ of unary operations on $M$, satisfying the conditions

and

$$
\begin{aligned}
x(r s) & =(x r) s \\
x(r+s) & =x r+x s \text { for all } x \in M, \text { all } r, s \in R \\
(x+y) s & =x s+y s \text { for all } x, y \in M, s \in S .
\end{aligned}
$$

Morphisms between $S$-distributive $R$-Modules are just homomorphisms in the sense of universal algebra, that is group homomorphisms which preserve the "scalar" multiplication by the elements of $R$. Note that the $S$-distributive $R$-modules form an equational class.

If $R$ has a unit, 1 , and $x 1=x$ for all $x \in M$ then $M$ is called unitary. An $R$-module is a $D R$-distributive $R$-module.

Evidently every near-ring $R$ can be given the structure of an $R$-module (unitary if $R$ is) by right multiplication in $R$. On the other hand, every group $G$ has a natural $T(G)$-module structure, given by applying the $f \in T(G)$ to the $s \in G$.

If a near-ring $R$ is actually a ring with unit 1 , then $D R=R$, and for each unitary $R$-module $M, x 2=x(1+1)=x+x$ for all $x \in M$ and so $x+y+x+y=$ $(x+y)(2)=x 2+y 2=x+x+y+y$ for all $x, y \in M$, which implies that the underlying group of $M$ is commutative. Thus the present notion of unitary 
$R$-module coincides with the usual one whenever $R$ is a ring with unit (Fröhlich [6]).

2. The semigroup near-ring $N[A]$. In this section we investigate the concept of the semigroup near-ring, first introduced in Fröhlich [6] by means of a construction which differs slightly from that presented here.

For a semigroup $A$, let $F$ be the free group over the underlying set of $A$, with + for the operation, and define a further binary operation $(s, t) \leadsto s t$ in $F$ as follows: For $s \in F$ and $a \in A$, let $s a=h_{a}(s)$, where $h_{a}$ is the endomorphism of $F$ mapping $x \leadsto x a(x \in A)$, and then put

$$
s\left(\varepsilon_{1} a_{1}+\cdots \varepsilon_{n} a_{n}\right)=\varepsilon_{1} s a_{1}+\cdots+\varepsilon_{n} s a_{n}
$$

for $a_{1}, \ldots, a_{n} \in A, \varepsilon_{i} \in\{1,-1\}$, where $\varepsilon_{1} a_{1}+\cdots+\varepsilon_{n} a_{n}$ is in reduced form, i.e. $\varepsilon_{i} a_{i} \neq-\varepsilon_{i+1} a_{i+1}$ for $i<n$. Note that this requirement can actually be dropped since a cancellation on the left, say $\varepsilon_{i} a_{i}=-\varepsilon_{i+1} a_{i+1}$, produces the analogous cancellation $\varepsilon_{i} s a_{i}=-\varepsilon_{i+1} s a_{i+1}$ on the right. It follows that $s(t+u)=s t+s u$ for all $s, t, u \in F$. Also, $s(-t)=-s t$ since $s(-t)+s t=s(-t+t)=s 0=0$, the latter equality being implicit in $\left(^{*}\right)$.

The resulting algebraic system is a near-ring $N[A]$, unitary if $A$ has a unit, +being its addition and $\left(^{*}\right)$ defining its multiplication. The latter evidently extends the multiplication on $A$ so that $A$ is a subsemigroup of the multiplicative semigroup of $N[A]$. Note that the elements of $A$ are distributive since the $h_{a}$ are endomorphisms of the additive group of $N[A]$ (which is just $F$ ).

In some sense, the near-ring $N[A]$ is for near-rings what the semigroup ring $Z[A]$ is for rings (Fröhlich [6]):

If $N$ is any near-ring then any multiplicative homorphism $h: A \rightarrow N$ such that all $h(a), a \in A$, are distributive elements of $N$ uniquely extends to a nearring homomorphism $f: N[A] \rightarrow N$. In addition, if $A$ and $N$ are unitary, and $h$ preserves units, then $f$ also does.

In the following, we will be interested in the distributive elements of certain $N[A]$. If $a \in A$ is a right zero (i.e. $c a=a$ for all $c \in A$ ) then for all $s=$ $\varepsilon_{1} a_{1}+\cdots+\varepsilon_{n} a_{n} \in N[A]$, where $a_{i} \in A, \quad \varepsilon_{i} \in\{1,-1\}, \quad s a=\varepsilon_{1} a+\cdots+\varepsilon_{n} a=$ $\left(\varepsilon_{1}+\cdots+\varepsilon_{n}\right) a=n_{s} a$ where $n_{s}$ is the "length" of $s$, i.e. the image of $s$ under the group homomorphism $N[A] \rightarrow Z$ which maps each element of $A$ to 1 . From this it follows immediately that $k a$ is distributive for all $k \in Z$.

Moreover, for a monoid $A$, the distributive elements of $N[A]$ are precisely the elements of $A$, and all elements $k a$ where $a \in A$ is a right zero and $k \in Z$. One sees this as follows: suppose $s=\varepsilon_{1} a_{1}+\cdots+\varepsilon_{n} a_{n}$ is distributive. Then

$$
\begin{aligned}
(1+1) s & =\varepsilon_{1} a_{1}+\cdots+\varepsilon_{n} a_{n}+\varepsilon_{1} a_{1}+\cdots+\varepsilon_{n} a_{n} \\
& =\varepsilon_{1} a_{1}+\varepsilon_{1} a_{1}+\cdots+\varepsilon_{n} a_{n}+\varepsilon_{n} a_{n}
\end{aligned}
$$

which yields $\varepsilon_{1} a_{1}=\varepsilon_{2} a_{2}=\cdots \varepsilon_{n} a_{n}(=\varepsilon a$ say), and so $s=\varepsilon n a$. If $\varepsilon=1=n$ then 
$s \in A$. If $\varepsilon=-1, n=1$ then for all $c \in A$,

$$
\begin{aligned}
(1+c) s & =(1+c)(-a)=-a-c a \\
& =-(1+c) a=-c a-a
\end{aligned}
$$

which yields $a=c a$ and so $a$ is a right zero. If $n \geq 2$ then for all $c \in A$,

$$
\begin{aligned}
(1+c) s & =\varepsilon a+\cdots+\varepsilon a+\varepsilon c a+\cdots+\varepsilon c a \\
& =\varepsilon a+\varepsilon c a+\cdots+\varepsilon a+\varepsilon c a
\end{aligned}
$$

and so $\varepsilon a=\varepsilon c a$, which implies that $a$ is a right zero.

The freeness property of $N[A]$ leads to a very convenient description of the category of $A$-distributive $N[A]$-modules for any semigroup $A$, and of the category of $N[A]$-modules if $A$ is a monoid. For any semigroup $A$, an $A$-group is a group $G$ together with an action of $A$ on $G$ by endomorphisms, i.e. unary operations $x \leadsto x a$ on $G$ for each $a \in A$ such that $(x y) a=(x a)(y a)$ and $x(a b)=$ ( $x a) b$, and an $A$-group homomorphism $f: G \rightarrow H$ is a group homomorphism compatible with the action of $A$, i.e. $f(x a)=f(x) a$. If $A$ has a unit 1 , then an $A$-group is unitary if in addition $x 1=x$ for all $x \in G$. A special A-group is an $A$-group $G$ such that $G a$ is abelian for all right zeroes $a \in A$.

It is clear that any $A$-distributive $N[A]$-module $G$ gives rise to an $A$-group (which is unitary if $A$ and $G$ are) by restricting the action of $N[A]$ to the multiplicative subsemigroup $A$ of $N[A]$.

Conversely, if $G$ is any $A$-group, the action of $A$ determines a multiplicative homomorphism from $A$ into the near-ring $T(G)$ such that the images of the elements of $A$ are distributive, and this extends to a near-ring homomorphism $N[A] \rightarrow T(G)$. The natural action of $T(G)$ on $G$ then provides an $A$ distributive $N[A]$-module structure on $G$ whose action extends the action of $A$ on $G$, and which is unitary if $A$ was unitary and $G$ was a unitary $A$-group. The resulting one-one correspondence between $A$-distributive $N[A]$-modules and $A$-groups evidently extends to the respective homomorphisms, the functors hereby defined giving a category isomorphism.

In analogous fashion, the category of $N[A]$-modules and the category of special $A$-groups are isomorphic if $A$ is a monoid. For any elements $s$ and $t$ of an $N[A]$-module $G$, and any rignt zero $a \in A$, one has $(s+t)(a+a)=$ $s(a+a)+t(a+a)$ since $a+a$ is a distributive element of $N[A]$, therefore $s a+t a+s a+t a=s a+s a+t a+t a$ and hence $t a+s a=s a+t a$, which shows $G a$ is abelian, i.e. the $A$-group determined by $G$ is special. Conversely, if $G$ is a special $A$-group then, for the associated $A$-distributive $N[A]$-module structure on $G$, one has

$$
(s+t)(k a)=k(s+t) a=k(s a+t a)=k s a+k t a
$$

for any right zero $a \in A, k \in Z$, and $s, t \in G$, the last step by the abelianness of $G a$. This shows $D N[A]$-distributivity. 
3. The Examples. The first example is the class of $\{1\}$-distributive unitary $Z$-modules, where $Z$ is the ring of integers. Obviously, $Z=N[A]$ for $A=\{1\}$, the one-element group. Thus, by the previous section, the category of $\{1\}$ distributive unitary $Z$-modules "is" the category of $\{1\}$-groups, and hence just the category of groups. It follows that there are no non-trivial absolute retracts (and therefore no non-trivial injectives) in this category, since every group can be embedded in a simple group. A recent proof for the latter (apparently longer known) result is contained in Boone-Higman [4]; actually, [4] deals only with the countable case, but this restriction is immaterial to the argument presented there.

This disproves the theorem in Seth-Tewari [9, p. 139] which claims a characterization of injectives analogous to the Baer characterization for modules over rings, i.e. that $A$ is injective iff any homomorphism $I \rightarrow A, I$ any right ideal of $R$, extends to a homomorphism $R \rightarrow A$. Any divisible abelian group, as unitary $\{1\}$-distributive $Z$-module, clearly satisfies the latter condition and thus provides a counterexample. We note that essentially the same counterexample has also been given by Oswald [9]. For the more restricted case of unitary $R$-modules ( $D R$-distributive!), we do not know whether the theorem is true or false.

Incidentally, one might wonder what the situation is if the restriction that the modules be unitary is dropped. That there is no miraculous improvement can be seen as follows: Suppose $M$ is an injective $\{1\}$-distributive $Z$-module (not necessarily unitary). Then the map $x \leadsto x \cdot 1$ is a retraction of $M$ onto the submodule $M \cdot 1$, and hence $M \cdot 1$ is also injective. But $M \cdot 1$ is a unitary module and hence trivial, by the above remarks. Consequently, $x \cdot n=0$ for all $x \in M, n \in Z$. Now, if $G \supseteq H$ are groups, and $h: H \rightarrow M$ is a group homomorphism then $G$ and $H$ can be made into $\{1\}$-distributive $Z$-modules by defining the action of $Z$ to be trivial (as it is in $M$ ), and then the injectivity of $M$ as module implies that there is an extension of $h$ to a homomorphism $G \rightarrow M$. This says the underlying group of $M$ is injective, and hence $M$ is trivial. Thus there are no non-trivial injective $\{1\}$-distributive $Z$-modules, either.

The second example shows the existence of unitary near-rings $R$ such that the category of $\boldsymbol{R}$-modules has no non-trivial absolute retracts. This is the case for any $R=N[A]$ where $A$ is a non-trivial group. By the results in the last section we only have to show that there are no non-trivial absolute retract unitary $A$-groups.

Suppose $G$ is such an absolute retract. Let $H$ be the semi-direct product $A \rtimes G$ whose elements are the pairs $(a, s), a \in G$, and whose multiplication is given by

$$
(a, s)(b, t)=(a b,(s b) t)
$$

Now, take any proper extension $S \supseteq H$ of $H$ by a simple group $S$ and make $A$ 
act (unitarily) on $S$ by $x a=(a, 1)^{-1} x(a, 1)$. Since $(a, 1)^{-1}(1, s)(a, 1)=(1, s a)$ for any $s \in G$, the map $s \leadsto(1, s)$ is then an $A$-group embedding $G \rightarrow S$, and therefore has a left inverse $h: S \rightarrow G$. $S$ being larger than $H, h$ cannot be one-one; by simplicity, this makes $h$, and therefore $G$, trivial.

Finally, we offer an example of a unitary near-ring $N$ such that not every unitary $N$-module has an injective extension, but nevertheless there are some non-trivial injective unitary $N$-modules, in fact, one for each injective abelian group. We take for $N$ the semigroup near-ring $N[A]$, where $A$ is any non-trivial monoid with zero element a (i.e. $a$ is both a left and a right zero). Note that in this case there are no other right zeroes in $A$ except $a$, and so the special $A$-groups are exactly those $A$-groups $G$ with $G a$ abelian. Consequently, any abelian group determines a special $A$-group by taking $A$ to act identically. We will show that the divisible abelian groups provide injectives in the category $\mathbf{S}$ of all special $A$-groups in this fashion. Let $D \in \mathbf{S}$ be of this kind and consider A-group homomorphisms $f: G \rightarrow H$ and $g: G \rightarrow D$ for any $G$, $H \in S$, where $f$ is an embedding. Then $g \mid G a: G a \rightarrow D$ and the map $h: G a \rightarrow H a$ induced by $f$ are homomorphisms between abelian groups, $h$ also an embedding. Since $D$ is injective as abelian group, one then has a group homomorphism $q: H a \rightarrow D$ such that $q h=g \mid G a$, and it readily follows that the map $x \leadsto q(x a)$ is an $A$-group homomorphism $p: H \rightarrow D$ for which $g=p f$. This shows $D$ is injective.

Note that, for any injective $G$ in $\mathbf{S}, G a$ is a retract of $G$ in $\mathbf{S}$ (the retraction being the effect of $a$ ) and hence also injective in $\mathbf{S}$; the abelian $D \in \mathbf{S}$ on which $a$ acts identically then show that $G a$ is a divisible abelian group. In particular, if $G=G a$ then $G$ itself is a divisible abelian group, which shows the converse of what is proved above.

It remains to show that not every $N[A]$-module has an injective extension; in fact, as promised in the introduction, we show that, for every non-trivial monoid $A$, the category of all $N[A]$-modules (or rather, what is equivalent, the category of all special $A$-groups) is not residually small. This is accomplished by showing that every group can be embedded in (the underlying group of) a special $A$-group; if the category of all special $A$-groups were residually small then every special $A$-group would have an equationally compact extension (see Taylor [10] or Banaschewski-Nelson [2]); the underlying group of an equationally compact $A$-group being an equationally compact group, this would imply that every group has an equationally compact extension, and hence that the category of groups is residually small, which contradicts the existence of arbitrarily large simple groups [10].

For any group $G$, define groups $G_{t}(t \in A)$ as follows: $G_{t}=G$ if $t \notin I$, and $G_{t}=G / G^{\prime}$ if $t \in I$, where $G^{\prime}$ is the commutator subgroup of $G$ and $I$ is the right ideal of $A$ generated by the right zeroes, i.e. $I=\{a t \mid a, t \in A, a$ a right zero $\}$. For $s, t \in A$, let $f_{t, s}: G_{t} \rightarrow G_{t s}$ be the identity map if $G_{t}=G_{t s}$, and the quotient map 
$G \rightarrow G / G^{\prime}$ otherwise. Let $K=\amalg G_{t}(t \in A)$ be the coproduct in the category of groups with natural injections $j_{t}: G_{t} \rightarrow K$, and for each $s \in A$, let ( ) $s: K \rightarrow K$ be the map such that ( )s $j_{t}=j_{t s} f_{t, s}$. Since $f_{t, r s}=f_{t r, s} f_{t, r}$ for all $r, s, t \in A$, this defines an action of $A$ on $K$. Moreover, if $a \in A$ is a right zero then $K a=\operatorname{Im} j_{a}=G / G^{\prime}$ and hence is abelian, so in this way $K$ actually becomes a special $A$-group. Since $A$ is non-trivial, $1 \notin I$, and thus $j_{1}$ provides the desired embedding of $G$ into a special $A$-group.

This evidently leaves open the following problem: Is there any distributively generated near-ring $N$ which is not a ring for which the category of all $N$-modules has enough injectives, or is residually small?

\section{REFERENCES}

1. B. Banaschewski, Injectivity and Essential Extensions in Equational Classes of Algebras. Proc. Conference on Universal Algebra (1969) Queen's Series in Pure and Applied Mathematics No. 25, Kingston, Ontario 1970.

2. B. Banaschewski and E. Nelson, Equational Compactness in Equational Classes of Algebras. Alg. Univ. 2 (1972), 152-165.

3. J. C. Beidleman, Quasi-regularity in near-rings. Math. Zeit. 89 (1965), 224-229.

4. W. W. Boone and G. Higman, An algebraic characterization of groups with soluble word problem. J. Aust. Math. Soc. 18 (1974), 41-53.

5. A. Fröhlich, Distributively generated Near-Rings I and II. Proc. London Math. Soc. 8 (1958), 76-108.

6. A. Fröhlich, On Groups over a d.g. near-ring (I), Quart, J. Math. 11 (1960), 193-210.

7. N. Jacobson, The Theory of Rings. New York. 1943.

8. A. Oswald, A note on injective modules over a d.g. near-ring. Canadian Math. Bull. (in print)

9. V. Seth and K. Tewari, On injective near-ring modules. Can. Math. Bull. 17 (1974), 137-142.

10. W. Taylor, Residually small varieties. Alg. Univ. 2 (1972), 33-53.

DEPARTMENT OF MATHEMATiCs

M'MASTER UNIVERSITY

Hamilton, Ont. Canada L8S 4K1 